\title{
Management of Parapneumonic Effusions in Pediatrics: Current Practice
}

\author{
Sarah C. McBride, MD \\ Children's Hospital Boston, Harvard Medical \\ School, Boston, Massachusetts.
}

\begin{abstract}
Pneumonia with associated complex pleural disease is a cause of significant morbidity among hospitalized children. The management of this patient population continues to be a challenge and varies even among single institutions. The article presented here reviews the management goals for pediatric patients hospitalized with complex parapneumonic effusions and provides updated summaries of both medical and surgical therapies. Journal of Hospital Medicine 2008;3:263-270. (C) 2008 Society of Hospital Medicine.
\end{abstract}

KEYWORDS: pediatrics, pneumonia, effusion, management guidelines.

D neumonia complicated by lung necrosis and pleural disease consisting of parapneumonic effusion or empyema is a cause of significant morbidity among pediatric inpatients. Current practice in caring for these patients is highly variable, even within single institutions. Medical management of hospitalized children with complex pneumonias includes an attempt to isolate the offending organism, tailored antibiotic therapy, and adequate pain management in association with pleural catheter drainage of large effusions. Thrombolytic agents are frequently trialed in an attempt to lyse loculated effusions without surgical intervention. Surgical drainage or decortication of walled-off infections is employed when there is poor response to more conservative treatment with pleural catheter drainage. The major therapeutic goal for this patient population is promotion of clinical recovery despite residual pleural abnormality at time of hospital discharge, with the knowledge that complete disease resolution is almost universal.

Variability in management as well as unpredictable patient response to differing therapeutic modalities has hindered the development of clear practice guidelines. Additionally, studies have suggested a shift in bacterial causative pathogens since the early 1990s, particularly after the heptavalent pneumococcal vaccine was added to routine childhood immunization schedules in 2000 , and have warned of the growing prevalence of methicillinresistant Staphylococcus aureus (MRSA). ${ }^{1}$

This article reviews the management of pediatric patients hospitalized with complex parapneumonic effusions and summarizes current diagnostic and therapeutic modalities to offer an updated approach to clinical practice.

\section{METHODS}

This review was constructed after careful appraisal of data from recent pediatric studies on parapneumonic effusions. The sub- 
headings in the Results section summarize the findings from these published studies and address the changing epidemiology, diagnostic techniques, and management options for this patient population. Finally, the author's impressions of management challenges related to the existing variation in clinical practice and the absence of strong evidenced-based guidelines are presented.

\section{RESULTS}

\section{Changing Epidemiology?}

In the recent past, an increase in the incidence of complicated pneumonia among pediatric patients has been reported, from 1993 to 2000, along with an increasing rate of drug-resistant pathogens. ${ }^{1-4}$ In the early 1990s Streptococcus pneumoniae (S. pneumoniae) was by far the most common etiologic agent of pneumonia with complicated parapneumonic effusions in the US, with most strains (70-75\%) susceptible to penicillins. ${ }^{1,3,5}$ However, after the widespread use of the pneumococcal conjugate vaccine in 2000 , studies began to report an increasing proportion of patients with complicated parapneumonic effusions resulting from Staphylococcus aureus (S. aureus), with a concerning increase in community-acquired methicillin-resistant strains (CA-MRSA). ${ }^{1}$ S. pneumoniae remains the most common causative bacterial pathogen in pediatric pneumonia as well as in complicated cases with pleural disease; however, a shift in trend toward more cases of $S$. aureus seems likely as more cases of CA-MRSA are reported among pediatric patients.

Historically, patients with more complex pleural disease tend to be slightly older (mean age 4-6 years), have a longer duration of fever prior to presentation (3-5 days), and are more likely to complain of chest pain on initial presentation compared with patients with uncomplicated pneumonia. ${ }^{2}$ There does not seem to be a sex preference for complex disease. Despite increasing concern about drug-resistant bacterial pathogens, patients with disease caused by drug-resistant organisms have been found to not have significantly worse disease on presentation or in clinical course compared with patients infected with drug-susceptible organisms. ${ }^{2,3,5}$

\section{Initial Evaluation}

A careful history can provide valuable clues to a patient's diagnosis of parapneumonic effusion.
TABLE 1

Evaluation of Patients with Suspected Parapneumonic Effusion

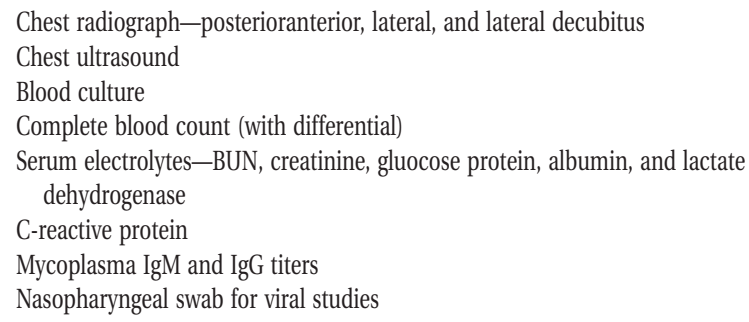

After initial assessment of airway, breathing, and circulation, focusing on a further workup for pulmonary processes and pleural disease is indicated (Table 1). Infectious signs and symptoms, often with localization to the chest, are present in the early stages of disease and become more obvious with larger effusions. Fever, increased work of breathing, cough, and shortness of breath as well as decreased breath sounds on the affected side and dullness to percussion are present in most cases once disease has progressed. A posteroanterior and lateral chest radiograph generally reveals either pneumonia with associated effusion or opacification of the hemithorax consistent with a large effusion with associated parenchymal infiltrate. A lateral chest radiograph can help to distinguish pleural disease from parenchymal disease, and a lateral decubitus film can help in the determination of whether pleural fluid is mobile. The volume of pleural fluid necessary for detection of an effusion in a posteroanterior radiograph is at least approximately $200 \mathrm{~mL}$ compared with only 10 to $50 \mathrm{~mL}$ in a lateral decubitus radiograph.

Once a plain radiograph detects an effusion of significant size or there is concern about loculation based on the lateral decubitus views, ultrasound is the subsequent diagnostic study of choice. Ultrasound has the ability to detect loculations in the pleural collection as well as solid lesions in the pleural space and can be used simultaneously as guidance for thoracentesis. Importantly, ultrasound is actually superior diagnostically to computed tomography (CT) in visualizing pleural loculations. However, CT is the preferred modality for imaging lung parenchyma and is indicated when a lung abscess is suggested by the initial imaging. Additionally, if malignancy is suspected, a CT is indicated.

As with other diagnoses in pediatrics, excluding other (noninfectious) causes of pleural effu- 
sion is important during evaluation. A history of renal or cardiac disease should raise concern for fluid overload situations. Signs or symptoms to suggest a more indolent progression of disease may indicate underlying malignancy or atypical infectious agents such as tuberculosis. Associated rheumatologic symptoms such as rashes or joint symptoms should also bring a diagnosis of primary infectious effusion into question. Similarly, a lack of parenchymal disease associated with an effusion, assessed by either plain radiograph or, in patients who have CT, as part of their evaluation, is unusual, and therefore other potential causes of pleural effusions should be considered.

In addition to chest imaging, other laboratory tests should include a blood culture (including anaerobes), sputum culture when attainable, a complete blood count and electrolytes (to evaluate for inappropriate antidiuretic hormone secretion syndrome), serum albumin, and C-reactive protein (helpful to follow serially in assessing response to therapy). Mycoplasma IgM and IgG titers are appropriate for patients in higher-risk age groups. An anterior nasal swab for methicillin-resistant $S$. aureus colonization and a nasopharyngeal swab for viral studies may also reveal potential disease pathogens.

\section{Staging of Pleural Effusions}

Pleural fluid associated with pneumonia progresses through stages related to the inflammatory process triggering its accumulation. The initial staging of pleural disease is important in guiding management decisions on admission.

- Stage 1-exudative stage: pleural fluid that is inflammatory in nature by definition and generally has a higher white blood cell (WBC) count, lactate dehydrogenase (LDH), and protein level with lower $\mathrm{pH}$ and glucose values than a transudative fluid.

- Stage 2-fibropurulant stage: fibrin deposition in the pleural space that causes septation in the pleural fluid (loculations). The WBC count is higher than in a simple exudative effusion with the fluid having a thicker gross appearance, progressing to frank pus (empyema).

- Stage 3-organizing stage: the intrapleural strands of fibrin (loculations) thicken to become a solid "peel." Depending on their size and location in the pleural space, these solid areas of fibrinous peel may lead to significantly impaired lung function because of entrapment or create new pleural potential spaces that can wall off infection. At this final stage of pleural disease, spontaneous resolution often occurs with time. However, chronic empyema can also ensue.

\section{Ultrasound Staging}

Ultrasound can also be used effectively to stage pleural effusions. ${ }^{6}$

- Stage 1: echogenic fluid without septation.

- Stage 2: fibrinous septation of pleural fluid without the presence of a homogenous loculation.

- Stage 3: visualization of an organized, multiloculated empyema surrounded by a thick parietal rind with associated lung entrapment.

\section{Pleural Fluid Analysis}

Pleural fluid analysis has long been used to classify pleural effusions. The light criteria were developed for adults with pleural effusions to distinguish infectious fluid from noninfectious fluid, ${ }^{7}$ but their application to pediatric effusions has not been formally validated. There is little indication for routine aspiration of pleural fluid in pediatrics solely for laboratory analysis. Unlike in adults, nearly all effusions in children are parapneumonic and are managed with pleural catheter drainage once a patient is symptomatic. Therefore, in most cases, pleural fluid should be sent for analysis only after a decision is made to place a drainage catheter. Nevertheless, once the decision is made to place a pleural drain, collection of pleural fluid for analysis should be performed simultaneously and may be helpful in staging an effusion. Attempting to aspirate pleural fluid from a catheter after it has been placed is not recommended and is likely to yield inaccurate results.

A complete diagnostic evaluation from pleural fluid sampling is summarized in Table 2 and includes sending a gram stain and aerobic and anaerobic bacterial cultures as well as a differential cell count. The utility of biochemical analysis in distinguishing effusion from empyema for guidance in the management of uncomplicated parapneumonic effusions has been disputed. ${ }^{3,8}$ Nevertheless, pH, glucose, protein, albumin, and $\mathrm{LDH}$ are generally sent from the pleural fluid to gain a clearer picture of pleural disease stage. An additional infectious workup may include sending fluid for acid-fast bacilli culture, mycoplasma PCR, and $\mathrm{KOH}$ prep. If noninfectious etiologies are suspected, a 
TABLE 2

Pleural Fluid Analysis

Gram stain

Bacterial culture (aerobic and anaerobic)

Cell count (with differential)

Acid-fast bacilli culture

Mycoplasma PCR

$\mathrm{pH}$

Glucose

Protein

Albumin

Lactase dehydrogenase

2-3 $\mathrm{mL}$ additional fluid on ice to be held in lab for potential further analysis

Other studies might include: triglyceride, $\mathrm{KOH}$ prep, cytology, amylase, ANA, creatinine

triglyceride level, cytology, amylase, ANA, and creatinine may be performed on pleural fluid as well.

\section{Disease Management}

The initial management of complicated parapneumonic effusions is summarized in Table 3 and includes oxygen delivery for hypoxia, intravenous fluid hydration, and empirical antibiotic therapy, as well as consultation with an interventional radiology or surgical team to discuss possible drainage methods. A management algorithm is also provided in Figure 1.

\section{Antibiotic Therapy}

The patterns of prevalence of infectious agents that lead to pneumonia and pleural disease changes over time. As mentioned earlier, in the early 1990s Streptococcus pneumoniae was far and away the most common etiologic agent of pneumonia with complicated parapneumonic effusions in the United States, with most strains $(70 \%-75 \%)$ susceptible to penicillins. ${ }^{1,3,5}$ After the introduction of the pneumococcal conjugate vaccine, an increase in parapneumonic effusions resulting from Staphylococcus aureus (S. aureus) with a concerning increase in community-acquired methicillin-resistant strains was reported in 1 study from Tennessee. ${ }^{1}$ In addition to these 2 major causative organisms, Hemophilus influenzae, group A Streptococcus, S. pyogenes, and mycoplasma should be considered potential etiologic agents. A more complete list of potential pathogens is provided in Table 4.

Selecting empirical antimicrobial therapy in a hospitalized child with complicated pneumonia ideally takes into consideration local epidemiolog- ical data. In general, antibiotics active against S. pneumoniae, S. pyogenes, and S. aureus should be employed initially. It is prudent in areas where the rate of community-acquired methicillin-resistant $S$. aureus is high to strongly consider the use of clindamycin, understanding that some strains of $S$. aureus will initially show susceptibility to clindamycin but possess mutations that enable inducible clindamycin resistance. The generalized use of vancomycin should be avoided and reserved only for patients who are significantly ill or possess life-threatening allergies to other antibiotics. Ideally, antibiotic therapy is tailored appropriately based on positive blood or pleural fluid culture results after sensitivity testing is performed. Newer polymerase chain reaction (PCR) tests aimed at isolating disease pathogens from pleural fluid are on the horizon and may improve the ability to tailor antibiotic therapy during hospitalization.

Antibiotic therapy should be delivered intravenously until the patient shows clinical improvement and ideally until the patient is afebrile. At this point, an additional 1- to 3-week course of oral antibiotics is generally given, depending on the length of the intravenous course.

\section{Management Challenges}

There is considerable controversy regarding the initial inpatient procedural management of complex parapneumonic effusions. Simple pleural catheter drainage is likely to be adequate for treatment of exudative effusions without significant loculations. The effectiveness of fibrinolytic agents administered through pleural catheters in complex pleural effusions has been disputed. Published studies have not yielded consistent results and have all had limitations related to sample size or methods used for disease staging. ${ }^{9-12}$ Adverse reactions have been reported with intrapleural fibrinolytic use, including chest pain, fever, and occasionally bleeding from the catheter site. ${ }^{13,14} \mathrm{~A}$

TABLE 3

Initial Management of Parapneumonic Effusion

\footnotetext{
Oxygen delivery as indicated

Empiric antibiotic therapy

Intravenous fluid therapy as indicated

Analgesia

Antipyretics

Consultation with service to perform pleural drainage
} 


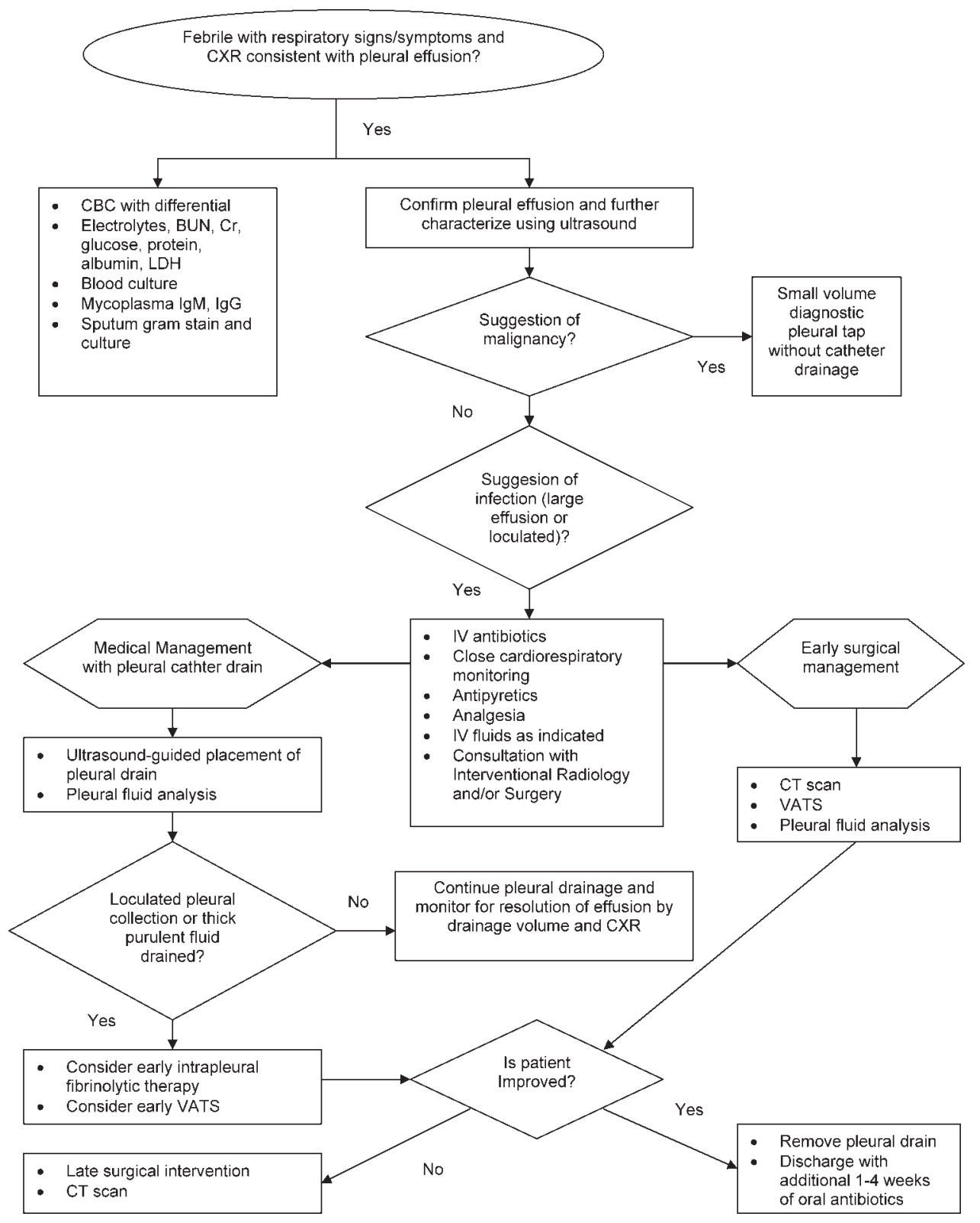

FIGURE 1. Parapneumonic effusion algorithm.

less invasive method of surgical intervention, termed video-assisted thoracoscopic surgery (VATS), has been employed for complex pleural effusions that have progressed to the organizational stage. This procedure enables direct visualization of the pleural space with the ability to lyse adhesions and drain fluid collections to afford optimal drainage. Thickened, hard pleural peels that cannot be removed using VATS require conversion to open thoracotomy.
Once a pleural catheter is placed and drainage begins to diminish with persistent radiographic evidence of effusion, fibrinolytic therapy can be administered in an attempt to break apart fibrin deposition to obtain free-flowing pleural fluid. The first randomized prospective trial comparing pleural catheter drainage with intrapleural urokinase to primary VATS for treatment of empyema in pediatric patients was recently carried out in London, United Kingdom, by Sonnappa et al. ${ }^{15}$ 
TABLE 4

Infectious Causes of Pleural Disease

Streptococcus pneumoniae

Staphylococcus aureus

Streptococcus pyogenes (group A streptococcus)

Haemophilus influenzae

Mycoplasma pneumoniae

Mycobacterium tuberculosis

Klebsiella pneumoniae

Pseudomonas aeruginosa

Escherichia coli

Anaerobes

Histoplasma capsulatum

Aspergillus

Nocardia asteroides

Coccidioides immitus

Legionella pneumophila

Among 60 hospitalized children with empyema, no significant difference in length of stay after intervention was found between the urokinase and VATS groups. Other secondary outcome measures were also found to be equivalent between the groups, including duration of pleural catheter drainage, total hospital length of stay, initial treatment failure, and resolution of disease by radiograph at 6-month follow-up. Urokinase is no longer available in the United States because of concerns related to viral contamination. Streptokinase is avoided because of its association with chest pain and fever. ${ }^{16}$ Most centers now employ tissue plasminogen activator (alteplase), a recombinant fibrinolytic with similar properties. A recent retrospective study of hospitalized children with parapneumonic effusions demonstrated slightly improved pleural drainage using alteplase compared with urokinase, with no systemic side effects or major complications. ${ }^{17}$ Alteplase can be administered once every 24 hours for a maximum of 3 doses.

Mobilization and ambulation are highly encouraged to prevent atelectasis and increase pleural catheter drainage. This necessitates adequate analgesia, often in the form of continuous infusions while a pleural catheter is in place. Chest physiotherapy is more likely to cause discomfort than to be beneficial to lung expansion in patients with complex pleural disease and therefore is not recommended.

Traditionally, clinical practice and earlier data have supported initial management of complex parapneumonic effusions with smaller-diameter pleural catheter ("pigtail") drainage. ${ }^{18}$ However, subsequent data suggested significantly shorter hospital length of stay and faster clinical improvement among patients treated more aggressively on admission with surgical procedures without reporting an increase in risk related to surgery or other complications. ${ }^{19-23}$ These studies had relatively small numbers of study subjects, and most did not control for disease stage at presentation. However, in cases of failed pleural catheter drainage, particularly after fibrinolytics have been attempted, surgery should be strongly considered in a persistently symptomatic patient. A chest CT scan is almost always performed prior to surgery to further evaluate the lung parenchyma and rule out lung abscesses, which generally should not be accessed because of the risk of introducing a fistulous tract. In an era in which hospital length of stay is a high priority and an important outcome measure, it is tempting to accept early surgical intervention as the new clinical practice standard based on existing studies. However, more information still needs to be gathered from larger-scale studies in order to draw this conclusion with confidence when there is a clear difference in the degree of invasiveness between these 2 management practices. In many centers sedation without general anesthesia is now used for pleural catheter placement, further delineating the difference in risk between simple pleural catheter drainage and surgical intervention.

\section{Outcome and Follow-up after Discharge}

Fortunately, most patients with complicated parapneumonic effusions have complete resolution of their disease with time. In the short term, diseaserelated complications include the development of lung abscess and bronchopleural fistula. Secondary scoliosis is commonly seen as well but is transient and resolves with resolution of the patient's underlying pulmonary process. ${ }^{24}$ Long-term complications are uncommon and related to persistent, mild restrictive lung defects. Even this complication is generally not clinically significant to cause limitations to activity and is only detected using pulmonary function tests. Essentially all radiographs are normal approximately 3-6 months after discharge. Follow-up with a pediatric pulmonologist is indicated whenever possible, particularly for severe cases. Patients with a remarkable history of past illnesses prior to hospitalization or with a protracted disease course 
should be evaluated for an underlying diagnosis affecting the immune system. This may include ruling out conditions capable of causing primary or secondary immune system impairment and cystic fibrosis.

\section{CONCLUSIONS}

Children with complicated parapneumonic effusions raise a challenge to pediatric hospitalists in choosing an initial management plan that is likely to be successful for their pleural disease stage on admission and to prevent the need for unnecessary intervention. Ultrasound is generally sufficient in diagnosing the stage of pleural disease and avoids both sedation and radiation exposure. It can also be used for guidance to access the pleural space effectively and position pleural catheters in the optimal location for maximum fluid drainage.

Clinicians must appreciate the degree of inflammation possible leading to pleural disease and the length of time necessary for complete disease resolution. Measures to keep patients comfortable and as mobile as possible during hospitalization, especially with pleural drains in place, coupled with proactive assessment of clinical response to initial therapy, are essential management goals.

Studies to compare hospital outcomes among patients receiving conservative medical management with antibiotics and pleural catheter drainage versus those undergoing early surgical debridement and drainage should be interpreted cautiously until larger studies are performed with attention to initial disease staging. Until that time, there is likely to be continued variability in practice even in an individual center because of factors related to hospitalist staff and surgical consultant staff impressions of initial illness and institutional resources to perform various procedures under conscious sedation versus general anesthesia at the time of admission.

Finally, it should be emphasized that disease pathogens should be restudied nationally to guide empirical antibiotic therapy because the treatment duration is longer than in most pediatric illnesses and patients may be at higher risk for adverse events related to prolonged antibiotic exposure. Further studies using large numbers of subjects from geographically diverse regions with more current epidemiological data are our best chance at defining the present picture of bacterial pathogens causing complicated pediatric pneumonia.

Address for correspondence and reprint requests: Sarah C. McBride, Children's Hospital Boston, Department of Medicine, 300 Longwood Avenue, Main Building, 9th floor, South Boston, MA 02012; E-mail: sarah.mcbride@ childrens.harvard.edu

Received 2 August 2007; revision received 30 0ctober 2007; accepted 5 November 2007.

\section{REFERENCES}

1. Buckingham SC, King MD, Miller ML. Incidence and etiologies of complicated parapneumonic effusions in children, 1996 to 2001. Pediatr Infect Dis J. 2003;22:449-504.

2. Byington CL, Spencer LY, Johnson TA, et al. An epidemiological investigation of a sustained high rate of pediatric parapneumonic empyema: risk factors and microbiological associations. Clin Infect Dis. 2002;34:434-440.

3. Tan TQ, Mason EO, Wald ER, et al. Clinical characteristics of children with complicated pneumonia caused by Streptococcus pneumoniae. Pediatrics. 2002;110:1-6.

4. Schultz KD, Fan LL, Pinsky J, et al. The changing face of pleural empyemas in children: epidemiology and management. Pediatrics. 2004;113:1735-1740.

5. Hardie WD, Roberts NE, Reising SF, Christie CDC. complicated parapneumonic effusions in children caused by penicillin-nonsusceptible Streptococcus pneumoniae. Pediatrics. 1998;101:338-392.

6. Light RW. A new classification of parapneumonic effusions and empyema. Chest. 1995;108:299-301.

7. Light RW, Macgregor MI, Luchsinger PC, Ball WC Jr. Pleural effusions: the diagnostic separation of transudates and exudates. Ann Intern Med. 1972;77:507-513.

8. Barnes NP, Hull J, Thomson AH. Medical management of parapneumonic pleural disease. Pediatr Pulmonol. 2005;39: 127-134.

9. Ozcelike D, Inci I, Nizam O, Onat S. Intrapleural fibrinolytic treatment of multiloculated postpneumonic pediatric empyemas. Ann Thorac Surg. 2003;76:1849-1853.

10. Maskell NA, Davies CWH, Nunn AJ, et al. U.K. controlled trial of intrapleural streptokinase for pleural infection. $N$ Engl J Med. 2005;352:865-874.

11. Tokuda Y, Matsushima D, Stein GH, Miyagi S. Intrapleural fibrinolytic agents for empyema and complicated parapneumonic effusions: a meta-analysis. Chest. 2006;129: 783-790.

12. Weinstein M, Restrepo R, Chait P, Connolly B, Temple M, Macarthur C. Effectiveness and safety of tissue plasminogen activator in the management of complicated parapneumonic effusions. Pediatrics. 2004;113:182-185.

13. Yao CT, Wu JM, Liu CC, Wu MH, Chuang HY, Wang JN. Treatment of complicated parapneumonic pleural effusion with intrapleural streptokinase in children. Chest. 2004;125: 566-571.

14. Thompson AH, Hull J, Kumar MR, Wallis C, Balfour Lynn IM. Randomized trial of intrapleural urokinase in the treatment of childhood empyema. Thorax. 2002;57:343347. 
15. Sonnappa S, Cohen G, Owens C, et al. Comparison of urokinase and video-assisted thoracoscopic surgery for treatment of childhood empyema. Am J Respir Crit Care Med. 2006;174:221-227.

16. Aye RW, Froese DP, Hill LD. Use of purified streptokinase in empyema and hemothorax. Am J Surg. 1991;161:560562.

17. Wells R, Havens P. Intrapleural fibrinolysis for parapneumonic effusion and empyema in children. Radiology. 2003; 228:370-378.

18. McLaughlin FJ, Goldmann DA, Rosenbaum DM, Harris GBC, Schuster SR, Strieder DJ. Empyema in children: clinical course and long-term follow-up. Pediatrics. 1984;73: 587-593.

19. Avansino JR, Goldman B, Sawin R, Flum DR. Primary operative versus nonoperative therapy for pediatric empyema: a meta-analysis. Pediatrics. 2005;115:1652-1659.
20. Subramaniam R, Joseph VT, Tan GM, Goh A, Chay OM. Experience with video-assisted thoracoscopic surgery in the management of complicated pneumonia in children. J Pediatr Surg. 2001;36:316-319.

21. Kercher KW, Attorri RJ, Hoover JD, Morton D. Thoracoscopic decortication as first-line therapy for pediatric parapneumonic empyema: a case series. Chest. 2000;118: 24-27.

22. Stovroff M, Teague F, Heiss KF, Parker P, Ricketts R. Thoracoscopy in the management of pediatric empyema. $J$ Pediatr Surg. 1995;30:1211-1215.

23. Kalfa N, Allal H, Lopez M, et al. Thoracoscopy in pediatric pleural empyema: a prospective study of prognostic factors. J Pediatr Surg. 2006;41:1732-1737.

24. Mukherjee S, Langroudi B, Rosenthal M, Balfour-Lynn IM. Incidence and outcome of scoliosis in children with pleural infection. Pediatr Pulmonol. 2007;42:221-224. 